\title{
Digitally Alloyed Modulated Precursor Flow Epitaxial Growth of Ternary AIGaN with Binary AIN and GaN Sub-Layers and Observation of Compositional Inhomogeneity
}

\author{
HEE JIN KIM, ${ }^{1}$ SUK CHOI,${ }^{1}$ DONGWON YOO, ${ }^{1,2,4}$ JAE-HYUN RYOU, ${ }^{1,5}$ \\ MICHAEL E. HAWKRIDGE ${ }^{3}$ ZUZANNA LILIENTAL-WEBER, ${ }^{3}$ \\ and RUSSELL D. DUPUIS ${ }^{1,2}$ \\ 1.-Center for Compound Semiconductors and School of Electrical and Computer Engineering, \\ Georgia Institute of Technology, 777 Atlantic Dr. NW, Atlanta, GA 30332-0250, USA. 2.-School \\ of Materials Science and Engineering, Georgia Institute of Technology, Atlanta, GA, USA. \\ 3.-Materials Science Division, Lawrence Berkeley National Laboratory, Berkeley, CA, USA. \\ 4.-Present address: Philips Lumileds Lighting, San Jose, CA, USA. 5.-e-mail: jaehyun.ryou@ \\ ece.gatech.edu
}

We report the growth of ternary aluminum gallium nitride (AlGaN) layers on AlN/sapphire template/substrates by digitally alloyed modulated precursor flow epitaxial growth (DA-MPEG), which combined an MPEG AlN sub-layer with a conventional metalorganic chemical vapor deposition (MOCVD)-grown $\mathrm{GaN}$ sub-layer. The overall composition in DA-MPEG $\mathrm{Al}_{x} \mathrm{Ga}_{1-x} \mathrm{~N}$ was controlled by adjustment of the growth time (i.e., the thickness) of the $\mathrm{GaN}$ sub-layer. As the $\mathrm{GaN}$ sub-layer growth time increased, the $\mathrm{Al}$ composition in $\mathrm{AlGaN}$ decreased to $50 \%$, but the surface morphology of the AlGaN layer became rough, and a three-dimensional structure with islands appeared for the DA-MPEG AlGaN with relatively thick GaN sub-layers, possibly resulting from the $\mathrm{Ga}$ adatom surface migration behavior and/or the strain built up from lattice mismatch between AlN and GaN sub-layers with increasing $\mathrm{GaN}$ sub-layer growth time. Through strain analysis by high-resolution x-ray diffraction, reciprocal space mapping, and scanning transmission electron microscopy, it was found that there was compositional inhomogeneity in the DA-MPEG AlGaN with AlN and GaN binary sub-layers for the case of the layer with relatively thick GaN sub-layers.

Key words: Aluminum gallium nitride (AlGaN), digitally alloyed modulated precursor flow epitaxial growth (DA-MPEG), metalorganic chemical vapor deposition (MOCVD)

\section{INTRODUCTION}

AlN and high aluminum content aluminum gallium nitride $(\mathrm{AlGaN})$ semiconductors have recently attracted much attention, due to their application in high-power and high-frequency electronic devices ${ }^{1}$ and deep ultraviolet optoelectronic devices. $^{2,3}$ For the realization of these devices, the epitaxial growth of high-quality AlN and AlGaN with high aluminum content is required. However, the

(Received July 22, 2009; accepted January 26, 2010; published online February 17, 2010) growth of such layers by conventional metalorganic chemical vapor deposition (MOCVD) (especially on a foreign substrate via strained heteroepitaxy) often suffers from a rough surface morphology and poor crystalline quality due to the lattice and thermal expansion mismatches between the $\mathrm{Al}_{x} \mathrm{Ga}_{1-x} \mathrm{~N}$ heteroepitaxial layer and the substrate, severe adduct formation between trimethylaluminum (TMAl) and ammonia $\left(\mathrm{NH}_{3}\right),{ }^{4}$ and limited migration (a short diffusion length) of adatoms (or surface species) of $\mathrm{Al}$ on a growing surface. High growth temperatures over $1300^{\circ} \mathrm{C}^{5,6}$ and low growth pressures of 
30-50 Torr $^{7}$ have been employed to enhance the surface migration of $\mathrm{Al}$ adatom species and to suppress parasitic gas-phase reactions (adduct formation) between $\mathrm{TMAl}$ and $\mathrm{NH}_{3}$. Increasing the growth temperature above $1300^{\circ} \mathrm{C}$ is, however, in many cases, restricted by the capability of the heating element of a reactor system, especially for resistive heating, and limited by low sticking coefficients of other column III elements such as gallium and indium at very high temperature for the growth of ternary or quaternary Al-containing alloys. An alternative way to achieve enhanced surface adatom migration and to improve the growth (minimize the parasitic reactions) is to use modulated precursor flow epitaxial growth (MPEG), which employs the separate introduction of the column III and column V precursors into the growth chamber in an alternating sequence. Similar growth schemes were demonstrated in InAs by MOCVD, ${ }^{8}$ AlN on sapphire substrates by MOCVD, ${ }^{9}$ and GaAs and AlGaAs by molecular beam epitaxy (MBE) ${ }^{10}$ We have also demonstrated the growth of high-quality AlN layers on free-standing AlN substrates at a relatively low growth temperature, employing the MPEG scheme. ${ }^{11}$ The MPEG AlN layer on an AlN substrate showed the excellent crystalline quality of a very narrow x-ray diffraction (XRD) peak with the full width at half maximum of 36 arcsec and 61 arcsec for symmetric (002) and asymmetric (102) diffractions, as well as a very smooth surface without any surface features. It was elucidated that this MPEG method is very effective to grow high-quality 'binary' AlN layers with crystalline and surface morphology qualities that are less sensitive to the growth temperature. A similar growth method was adopted for 'ternary' $\mathrm{AlGaN}$ growth ${ }^{12}$ that is, the introduction of group III precursors [a mixture of TMAl and trimethylgallium (TMGa) with a certain ratio, targeting a certain solid alloy composition] separated from the $\mathrm{NH}_{3}$ input; however, we found that the MPEG method was not very effective for the growth of ternary $\mathrm{Al}_{x} \mathrm{Ga}_{1-x} \mathrm{~N}$ layers, which was mainly due to the significantly different sticking coefficients of $\mathrm{Al}$ and $\mathrm{Ga}$ adatoms at a temperature of $1050^{\circ} \mathrm{C}$ (lower than the conventional $\mathrm{AlGaN}$ growth temperature but high enough to induce an extremely low sticking coefficient of $\mathrm{Ga}$ adatoms without $\mathrm{NH}_{3}$ overpressure). As a result, it turned out to be very difficult to grow an AlGaN layer with an $\mathrm{Al}$ content of less than $90 \%$ by the MPEG method.

In this study, we explored another growth scheme, named digitally alloyed modulated precursor flow epitaxial growth (DA-MPEG), which combines an MPEG AlN sub-layer with a conventional MOCVD GaN sub-layer for the growth of a high-quality AlGaN layer. This growth scheme is designed to minimize the desorption of Ga adatoms on the surface, and the composition of the ternary $\mathrm{Al}_{x} \mathrm{Ga}_{1-x} \mathrm{~N}$ layer can be controlled by the ratio of the GaN sub-layer to the AlN sub-layer. We describe the growth and characteristics of the AlGaN layer with various $\mathrm{Al}$ compositions in the range of $x_{\mathrm{Al}}=0.5-1$ by the DA-MPEG method and the compositional inhomogeneity that occurs in a DA-MPEG AlGaN layer with a low $\mathrm{Al}$ composition $\left(x_{\mathrm{Al}} \approx 0.5\right)$.

\section{EXPERIMENTAL PROCEDURES}

DA-MPEG AlGaN layers were grown in a Thomas Swan Scientific Equipment MOCVD reactor system $\left(6 \times 2^{\prime \prime}\right.$ wafer loading capacity) on AlN/sapphire templates. EpiPure ${ }^{\mathrm{TM}}$ TMGa, TMAl, and $\mathrm{NH}_{3}$ were used for the group III and group V precursors. For the preparation of AlN/sapphire templates, a $900 \mathrm{~nm}$-thick AlN layer was grown on a (0001) sapphire substrate by standard two-step growth heteroepitaxy employing a low-temperature AlN buffer layer without employing a special growth scheme $^{13}$ to reduce the threading dislocation density of AlN templates. Therefore, the total dislocation density for these layers was considered to be high $\left(>10^{9} \mathrm{~cm}^{-2}\right)$, while the surface was confirmed to be free of surface features such as nano-pits. Then, DA-MPEG AlGaN layers were grown at a growth temperature of $T_{\mathrm{g}} \approx 1050^{\circ} \mathrm{C}$. All AlN and AlGaN layers were grown at a chamber pressure of 75 Torr. Figure 1 shows the schematic sequence and modulated precursor flows for the growth of the DA-MPEG AlGaN. The DA-MPEG AlGaN layer consisted of an MPEG AlN sub-layer and a conventional MOCVD-grown GaN sub-layer. For the growth of the first MPEG AlN sub-layer, the $\mathrm{Al}$ precursor with a flow rate of $12 \mu \mathrm{mol} / \mathrm{min}$ and $\mathrm{NH}_{3}$ with a flow rate of $0.046 \mathrm{~mol} / \mathrm{min}$ were separately introduced into the chamber to deposit a monolayerthick AlN sub-layer for $5.9 \mathrm{~s}$ and $11.2 \mathrm{~s}$, respectively. This process is considered to be self-limiting-the thickness per cycle of the AlN grown by MPEG corresponds to the thickness of a monolayer of AlN. Then, the conventional GaN sub-layer was subsequently grown to suppress Ga desorption with TMGa and $\mathrm{NH}_{3}$ flow rates of $130 \mu \mathrm{mol} / \mathrm{min}$ and $0.11 \mathrm{~mol} / \mathrm{min}$, respectively. To control the total alloy composition in the $\mathrm{Al}_{x} \mathrm{Ga}_{1-x} \mathrm{~N}$ layer, we adjusted the growth time (hence, the thickness) of the GaN

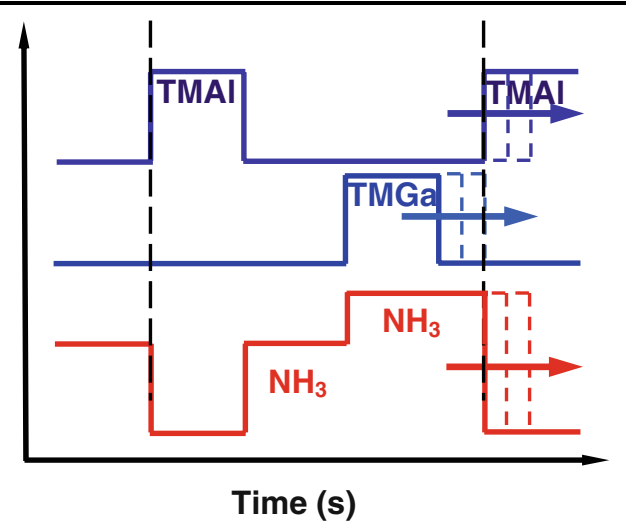

Fig. 1. Diagram showing flow rate modulation of the precursors of the DA-MPEG AIGaN layers. 
sub-layer from $9 \mathrm{~s}$ to $21 \mathrm{~s}$. The thickness of the DA-MPEG AlGaN layers was in the range of 50-70 nm, determined by transmission electron microscopy (TEM). Surface morphologies of the MPEG AlGaN layers were investigated in both macroscopic and microscopic scales by Nomarski optical microscopy, atomic force microscopy (AFM), and TEM. The crystalline quality and alloy composition of the AlGaN films were determined by highresolution x-ray diffraction (HR-XRD) rocking curves and reciprocal space mapping (RSM), and the optical property was investigated by optical transmittance measurement in the ultraviolet (UV) spectral region.

\section{RESULTS AND DISCUSSION}

The growth of ternary $\mathrm{Al}_{x} \mathrm{Ga}_{1-x} \mathrm{~N}$ layers with varying compositions by DA-MPEG with binary sub-layers was characterized by HR-XRD. Figure 2a shows the XRD (002) $\omega-2 \theta$ scan curves of the DA-MPEG AlGaN layers with various GaN sublayer growth times. The peak corresponding to the AlN layer in the template was observed at $\omega \approx 18.02^{\circ}$, together with the AlGaN peaks for all DA-MPEG AlGaN samples. For the DA-MPEG AlGaN layer with a GaN sub-layer growth time of $9 \mathrm{~s}$, the peak from the AlGaN was very close to the AlN peak and was not easily resolved. By Gaussian deconvolution, the peak of the DA-MPEG-grown AlGaN having a GaN sub-layer with a growth time of $9 \mathrm{~s}$ was estimated to be located at $17.99^{\circ}$. As the GaN sub-layer growth time was varied from $9 \mathrm{~s}$ to $21 \mathrm{~s}$, the peaks from the AlGaN layer shifted toward the lower Bragg angle side, from $17.99^{\circ}$ to $17.31^{\circ}$, indicating a higher Ga mole fraction in the layer, as shown in Fig. 2b. Furthermore, the satellite peaks were not observed, even with a wide range of $\omega-2 \theta$ scan with a $\omega$ scan range of $18^{\circ}$ (data not shown)-according to the XRD simulation, if the layer had grown with an AlN/GaN superlattice structure, each with a monolayer thickness, the satellite peaks should have appeared within 28,000 arcsec of the $\omega-2 \theta$ scan. It suggested that the DA-MPEG AlGaN layers had grown with intermixed interfaces, not with a well-defined superlattice structure; that is, the grown materials could be treated as single-phase $\mathrm{AlGaN}$ (except the DA-MPEG AlGaN with a GaN sub-layer growth time of $21 \mathrm{~s}$, as will be discussed later). From this XRD peak shift with increasing the GaN sub-layer growth time, it is clear that the overall $\mathrm{Al}$ composition $\left(x_{\mathrm{Al}}\right)$ in the DA-MPEG AlGaN layer can be controlled by adjustment of the GaN sub-layer growth time. The DA-MPEG AlGaN layer with a GaN sub-layer growth time of $21 \mathrm{~s}$, unlike other samples, appeared as double peaks corresponding to $\omega$ of $17.67^{\circ}$ and $17.31^{\circ}$, as shown in Fig. 2a. These double AlGaN peaks may suggest that this AlGaN layer consisted of $\mathrm{AlGaN}$ phases with two different $\mathrm{Al}$ compositions. One of the important characteristics of the group III nitride materials is their
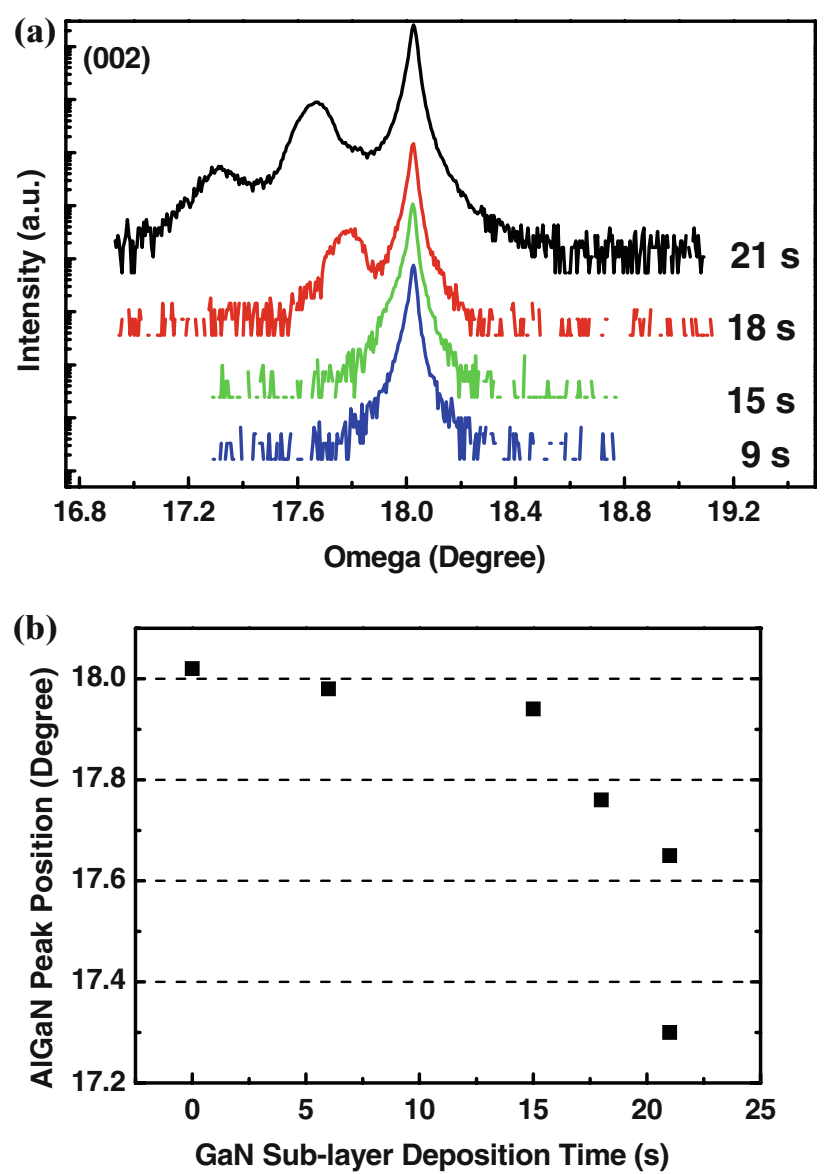

Fig. 2. (a) HR-XRD $\omega-2 \theta$ scans for (002) diffraction of the DA-MPEG AIGaN layers with GaN sub-layer growth times of $9 \mathrm{~s}$, $15 \mathrm{~s}, 18 \mathrm{~s}$, and $21 \mathrm{~s}$, and (b) XRD peak position of DA-MPEG AIGaN with GaN sub-layer growth time.

compositional inhomogeneity, which has also been reported as a compositional fluctuation, compositional modulation, or phase separation in InGaN materials ${ }^{14-16}$ and AlGaN materials. ${ }^{17}$ The compositional inhomogeneity has been well investigated in the low $\mathrm{Al}$ composition AlGaN on GaN, but it has been less studied in AlGaN with $\mathrm{Al}$ compositions higher than $50 \%$ on AlN. However, the double peaks in HR-XRD can be caused not only by compositional inhomogeneity but also by non-uniform strain relaxation beyond the critical thickness. ${ }^{18}$ The RSMs for (105) asymmetric diffraction were carried out to investigate the strain status of the DA-MPEG AlGaN layers with GaN sub-layer growth times of $18 \mathrm{~s}$ and $21 \mathrm{~s}$ grown on AlN templates. Figure $3 \mathrm{a}$ and $\mathrm{b}$ show a uniform contour around the peaks from the substrate and the layer, indicating a good crystalline quality. In Fig. 3 , the blue dotted line (i.e., a vertical line intersecting an AlN reciprocal lattice point having the same reciprocal lattice vector, $Q_{x}$, as AlN) represents a fully-strained (pseudomorphic) layer material, while the red dotted line (i.e., a line connecting the AlN reciprocal lattice point to a $\mathrm{GaN}$ reciprocal lattice point) represents a fully relaxed (having bulk lattice 

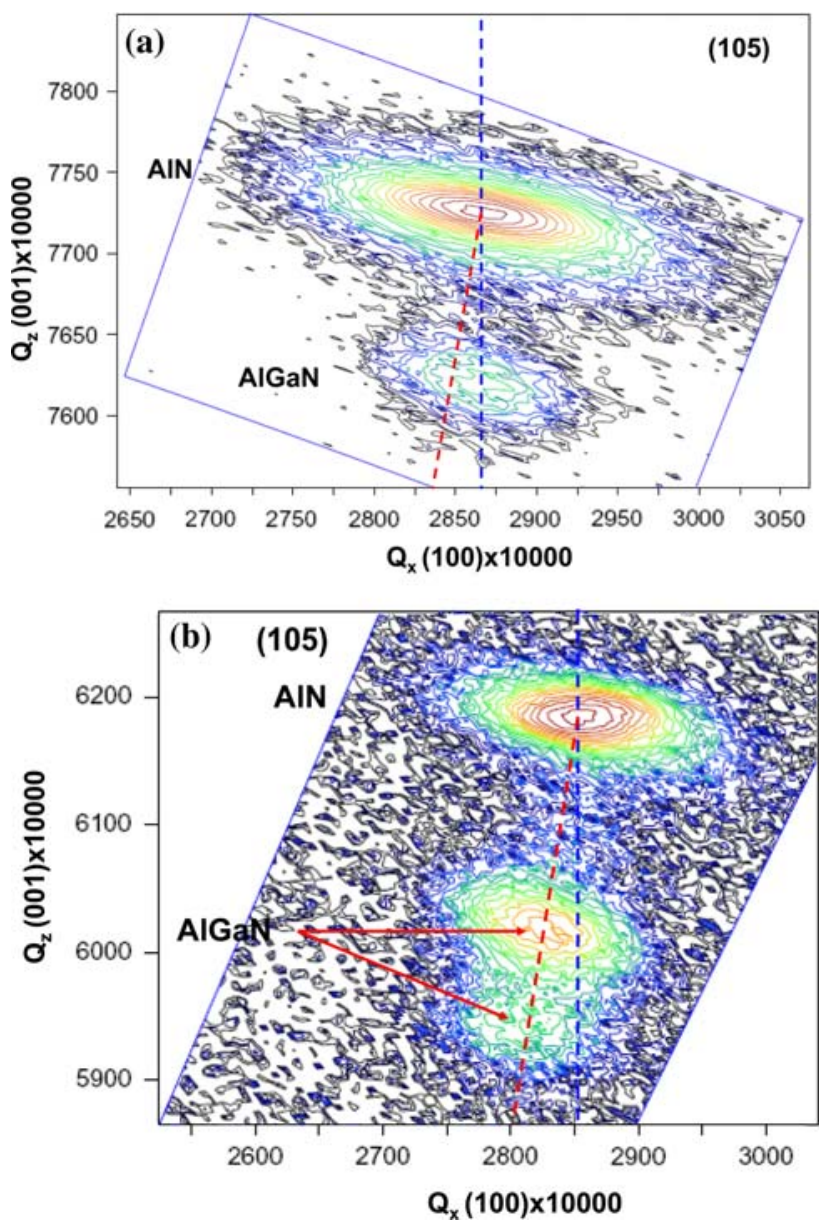

Fig. 3. RSMs for (105) diffraction of the DA-MPEG AIGaN layers with $\mathrm{GaN}$ sub-layer growth times of $18 \mathrm{~s}$ and $21 \mathrm{~s}$.

parameters) layer material. Because the lattice points for the AlGaN layer and the AlN layer are aligned along the vertical line, it is clear that the DA-MPEG AlGaN layer with a GaN sub-layer growth time of $18 \mathrm{~s}$ is fully strained to the AlN/ sapphire template, as shown in Fig. 3a. In addition, the other DA-MPEG AlGaN layers with growth times of $\mathrm{GaN}$ sub-layers less than $18 \mathrm{~s}$ are expected to be pseudomorphic. However, in the DA-MPEG AlGaN layer with a GaN sub-layer growth time of $21 \mathrm{~s}$, the position of the layer peak is shifted along the direction parallel to the surface, falling on the red dotted line, which indicates that it is relaxed from the AlN/sapphire template. Moreover, another peak from the AlGaN layer also appeared in the RSM, again shifted in a direction parallel to the surface. From the RSM peak position and Vegard's law, the Al composition in the DA-MPEG AlGaN was evaluated to be $x_{\mathrm{Al}}=75.05 \%$ for the $\mathrm{AlGaN}$ with a GaN sub-layer growth time of $18 \mathrm{~s}$ and $x_{\mathrm{Al}}=51.54 \%$ and $x_{\mathrm{Al}}=2.81 \%$ for the $\mathrm{AlGaN}$ with a GaN sub-layer growth time of $21 \mathrm{~s}$, respectively. It suggests that the double XRD peaks in the DA-MPEG AlGaN with a GaN sub-layer growth time of $21 \mathrm{~s}$ originated not from non-uniform strain

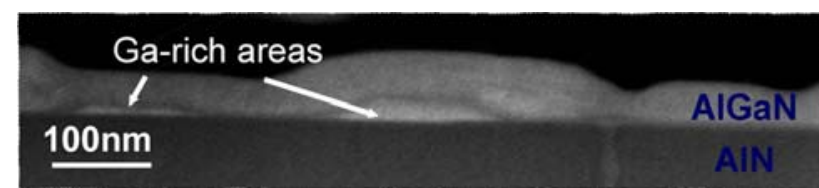

Fig. 4. z-Contrast scanning transmission electron microscopy (STEM) image of the DA-MPEG AIGaN layer with a GaN sub-layer growth time of $21 \mathrm{~s}$.

relaxation but from the compositional inhomogeneity in the $\mathrm{AlGaN}$ layer grown in this condition by DA-MPEG. The compositions of other DA-MPEG AlGaN layers with GaN sub-layer growth times of $9 \mathrm{~s}$ and $15 \mathrm{~s}$ were estimated to be $x_{\mathrm{Al}}=97.03 \%$ and $x_{\mathrm{Al}}=92.78 \%$, respectively.

The compositional inhomogeneity of the DA-MPEG AlGaN with a GaN sub-layer growth time of $21 \mathrm{~s}$ was also confirmed by $z$-contrast scanning TEM, as shown in Fig. 4. A Ga-rich area in the DA-MPEG AlGaN was found to be located under the large islands, while an Al-rich area was observed on a relatively flat surface (Fig. 4). Ga-rich areas also covered large regions and were on top of dislocations (data not shown here but in Ref. 19). This may be explained by the fact that the Ga adatoms generally have weaker bonding energy than that of the Al ad-atoms on the growing surface, which results in the larger surface migration length of the $\mathrm{Ga}$ adatoms than that of the $\mathrm{Al}$ adatoms. Especially, it could be accelerated in this DA-MPEG growth scheme because the Ga precursor was introduced to the chamber without the $\mathrm{Al}$ precursor; therefore, in this growth scheme, the Ga atoms have enough time to find the minimum energy site than if the conventional growth scheme is utilized. This phenomenon is similar to that reported for the InGaN growth, in which phase separation might be caused by the atomic size difference, i.e., bigger atom accumulation. ${ }^{20}$ The difference in surface migration lengths of the group III adatoms is expected to play an important role in the phase separation effect in AlGaN. Also, this may be accelerated around dislocations where the large numbers of dangling bonds and the $\mathrm{Ga}$ atoms stick at the dislocation core and are continuously enhanced with increasing $\mathrm{GaN}$ sub-layer growth time. In addition, an increase of the strain build-up with GaN sub-layer growth time may expedite the surface undulation by a StranskiKrastanow (S-K) growth mode ${ }^{21}$ that might contribute to this compositional inhomogeneity. When the GaN sub-layer growth time was increased to $21 \mathrm{~s}$, the growth of the GaN sub-layer experienced a transition from two-dimensional growth to threedimensional (3-D) growth on the AlN template. Once the S-K growth mode had started, the compositional fluctuation could occur, as observed in other ternary self-assembled quantum dots grown by S-K growth. ${ }^{22}$ This S-K growth of the repeated GaN sub-layers results in the surface undulation for the relaxation of accumulated strain. From XRD, it 

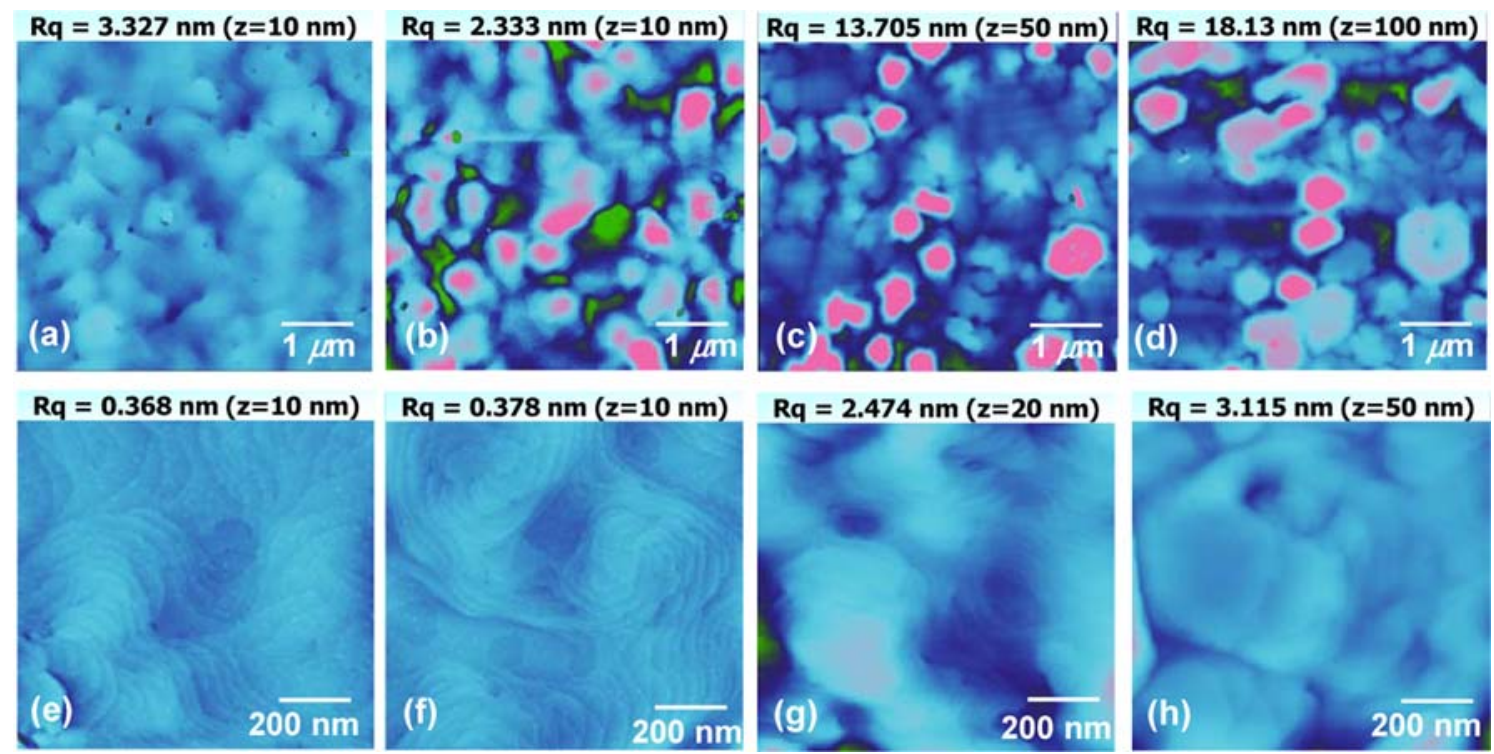

Fig. 5. Microscopic surface morphology of the DA-MPEG AIGaN layers measured by AFM of a $5 \times 5 \mu \mathrm{m}^{2}$ scan area grown on AIN/sapphire templates with GaN sub-layer growth times of (a) $9 \mathrm{~s}$, (b) $15 \mathrm{~s}$, (c) $18 \mathrm{~s}$, and (d) $21 \mathrm{~s}$, and of a $1 \times 1 \mu \mathrm{m}^{2}$ scan area with growth times of (e) $9 \mathrm{~s}$, (f) $15 \mathrm{~s}$, (g) $18 \mathrm{~s}$, and (h) $21 \mathrm{~s}$.

is considered that the relaxation of the strain also occurs via in-plane lattice parameter change. After the layers had relaxed via the Ga-rich region, an Al-rich AlGaN ternary layer was believed to have grown layer-by-layer, as can be seen in Fig. 4. Detailed TEM measurements have been reported elsewhere. ${ }^{19}$

The surface morphology of the DA-MPEG layers was explored by Nomarski microscopy in macroscopic scale and AFM in microscopic scale. Figure 5 shows the AFM images of DA-MPEG AlGaN samples with various $\mathrm{GaN}$ sub-layer growth times. The surface morphology of DA-MPEG AlGaN layers grown on the AlN/sapphire templates tended to be rougher with increasing growth time of the GaN sub-layer. The DA-MPEG AlGaN with a GaN growth time of $9 \mathrm{~s}$ showed a flat surface with a rootmean square (RMS) roughness of $1.231 \mathrm{~nm}$ and $0.368 \mathrm{~nm}$ for $5 \times 5 \mu \mathrm{m}^{2}$ and $1 \times 1 \mu \mathrm{m}^{2}$ scan areas, respectively, which was similar to that of typical AlN layers grown at high temperatures, ${ }^{11}$ as shown in Fig. 5a and e. As the GaN sub-layer growth time was increased to $15 \mathrm{~s}$, however, the RMS roughness dramatically increased and 3-D island structures began to appear. Further increase of the GaN sublayer growth time to $21 \mathrm{~s}$ enhanced 3-D growth, showing an RMS roughness of $18.13 \mathrm{~nm}$ and $3.115 \mathrm{~nm}$ for $5 \times 5 \mu \mathrm{m}^{2}$ and $1 \times 1 \mu \mathrm{m}^{2}$ scan areas, respectively, as shown in Fig. 5d and h. The size of the 3-D features also increased with GaN sub-layer growth time and reached an average height of $500 \mathrm{~nm}$ and an average width of $30 \mathrm{~nm}$ in the DA-MPEG AlGaN with a GaN sub-layer growth time of $18 \mathrm{~s}$. This change of surface morphology might have originated from the increase in accumulated strain with increasing GaN sub-layer growth time caused by lattice mismatch $(\sim 3.5 \%)$ between the AlN sublayer/template and the GaN sub-layer. Also, considering the surface morphology and XRD of the DA-MPEG AlGaN with the GaN sub-layer growth times of $15 \mathrm{~s}$ and $18 \mathrm{~s}$ in terms of S-K growth mode, that with the GaN sub-layer growth time of $15 \mathrm{~s}$ was thick enough to initiate the 3 -D growth mode while the layer was still fully strained. For the GaN sub-layer growth time of $18 \mathrm{~s}$, the relaxation of strain occurred via in-plane lattice constant change as well as 3-D growth mode due to a higher accumulated strain of the GaN sub-layer with a thicker layer.

In order to investigate the optical properties and bandgap of the DA-MPEG AlGaN layers with varying GaN sub-layer growth time, we measured the optical transmittance in a UV spectral regime (Fig. 6). The DA-MPEG AlGaN layers with GaN sub-layer growth times of $9 \mathrm{~s}$ and $15 \mathrm{~s}$ showed sharp cut-off wavelengths at $\sim 208 \mathrm{~nm}$ and $\sim 209 \mathrm{~nm}$, respectively. The photon which had an energy larger than the bandgap and was not fully absorbed by a thin DA-MPEG AlGaN layer appeared as a tail continuing to approximately $200 \mathrm{~nm}$. The sloped cut-off appeared at $\sim 236 \mathrm{~nm}$ and $\sim 279 \mathrm{~nm}$ for the DA-MPEG AlGaN with GaN sub-layer growth times of $18 \mathrm{~s}$ and $21 \mathrm{~s}$, respectively. The decrease of transmittance intensity in the sample with longer GaN sub-layer growth time came from the larger photon scattering at the rougher surface, which is shown in the AFM images (Fig. 5). Especially for the DA-MPEG with a GaN sub-layer growth time of $21 \mathrm{~s}$, the transmittance intensity started to decrease from $360 \mathrm{~nm}$. It was indirectly shown that there was compositional inhomogeneity in this sample. According to various suggested AlGaN bandgap 


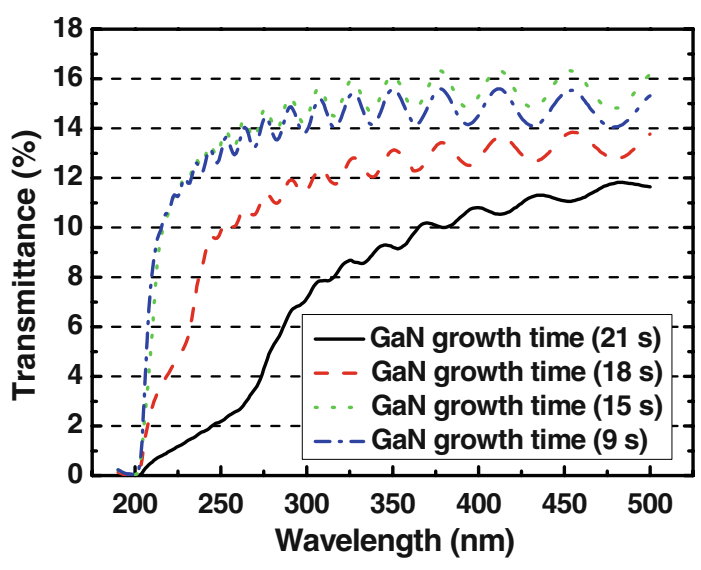

Fig. 6. Optical transmittance measurement for the DA-MPEG AIGaN layers with GaN sub-layer growth times of $9 \mathrm{~s}, 15 \mathrm{~s}, 18 \mathrm{~s}$, and $21 \mathrm{~s}$.

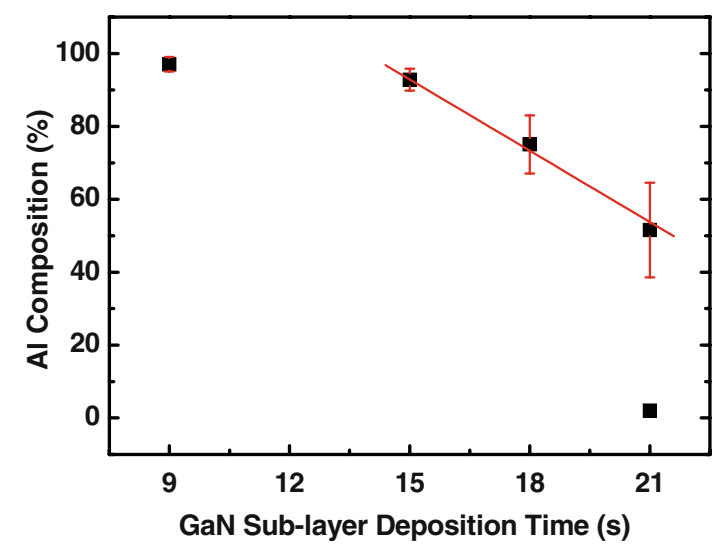

Fig. 7. The compositional change of the DA-MPEG AIGaN layers with the MPEG AIN and the conventional GaN binary sub-layers, depending on the growth time of the GaN sub-layer. Note that the error range of composition was obtained from optical transmittance measurements.

bowing parameters, ${ }^{23,24}$ the $\mathrm{Al}$ composition deduced from the cut-off wavelengths lies in the range of $x_{\mathrm{Al}}=0.93-0.95$ for the DA-MPEG AlGaN with a GaN sub-layer growth time of $9 \mathrm{~s}$, and $x_{\mathrm{Al}}=0.95-$ 0.92 for that of $15 \mathrm{~s}, x_{\mathrm{Al}}=0.80-0.72$ for that of $18 \mathrm{~s}$, and $x_{\mathrm{Al}}=0.59-0.46$ for that of $21 \mathrm{~s}$, which were well-matched with the $\mathrm{Al}$ compositions from the XRD measurement.

The relationship between $\mathrm{Al}$ composition in the DA-MPEG AlGaN layers and the growth time of the GaN sub-layer was evaluated and is shown in Fig. 7. The composition hardly changed from $97 \%$ when the GaN sub-layer growth times were shorter than $15 \mathrm{~s}$, however, linearly decreased to $50 \%$ after $15 \mathrm{~s}$, showing two distinct regions. This indicated that a 'latent' period for the initiation of $\mathrm{GaN}$ growth longer than $9 \mathrm{~s}$ existed in the $\mathrm{GaN}$ sub-layer growth; once the GaN growth had 'stabilized,' however, the thickness of the GaN was proportional to the growth time having the same growth rate. From the results, we found that the DA-MPEG method combined with a GaN sub-layer was effective in controlling $\mathrm{Al}$ composition in the $\mathrm{AlGaN}$ layer. However, compositional inhomogeneity appeared in the AlGaN layer with a long GaN sublayer growth time and low $\mathrm{Al}$ composition of $\sim 50 \%$. We believe that the control of threading dislocation density and the suppression of the initiation of 3-D growth induced by $\mathrm{S}-\mathrm{K}$ growth can mitigate the compositional inhomogeneity and rough surface morphology. To resolve this problem, another DA-MPEG method using an AlGaN sub-layer may be required, and the results will be reported elsewhere. ${ }^{25}$

\section{SUMMARY}

We described the digitally alloyed modulated precursor flow growth of high quality AlGaN with high $\mathrm{Al}$ content, employing precursor flow modulation for the MPEG AlN sub-layer and conventional GaN sub-layer. The overall alloy composition was controlled from $x_{\mathrm{Al}}=0.97$ to $x_{\mathrm{Al}}=0.50$ by adjustment of the GaN sub-layer growth time from $9 \mathrm{~s}$ to $21 \mathrm{~s}$. The surface morphology in DA-MPEG showed a clear tendency with $\mathrm{Al}$ composition in $\mathrm{AlGaN}$, and it became rough with 3-D island structures due to increased lattice mismatch with $\mathrm{GaN}$ sub-layer growth time. Double XRD peaks were observed from the DA-MPEG AlGaN with a GaN sub-layer growth time of $21 \mathrm{~s}$. Through detailed strain investigation by RSM and STEM analysis, it was confirmed that there was compositional inhomogeneity in DA-MPEG AlGaN. To improve the material's quality and surface morphology, suppression of the initiation of the 3-D growth in the sub-layer by conventional MOCVD may be required, which suggests that a smaller strain sub-layer may be needed.

\section{ACKNOWLEDGEMENTS}

R.D.D. acknowledges the support of the Steve W. Chaddick Endowed Chair in Electro-Optics and the Georgia Research Alliance.

\section{REFERENCES}

1. S. Arulkumaran, M. Sakai, T. Egawa, H. Ishikawa, T. Jimbo, T. Shibata, K. Asai, S. Sumiya, Y. Kuraoka, M. Tanaka, and O. Oda, Appl. Phys. Lett. 81, 1131 (2002).

2. Y. Bilenko, A. Lunev, X. Hu, J. Deng, T.M. Katona, J. Zhang, R. Gaska, M.S. Shur, W. Sun, V. Adivarahan, M. Shatalov, and M.A. Khan, Jpn. J. Appl. Phys. 44, L98 (2005).

3. Y. Taniyasu, M. Kasu, and T. Makimoto, Nature 441, 325 (2006).

4. C.H. Chen, H. Liu, D. Steigerwald, W. Imler, C.P. Kuo, M.G. Craford, M. Ludowise, S. Lester, and J. Amano, J. Electron. Mater. 25, 1004 (1996).

5. Y. Ohba and A. Hatano, Jpn. J. Appl. Phys. 35, L1013 (1996).

6. M. Imura, K. Nakano, T. Kitano, N. Fujimoto, N. Okada, K. Balakrishnan, M. Iwaya, S. Kamiyama, H. Amano, I. Akasaki, K. Shimono, T. Noro, T. Takagi, and A. Bandoh, Phys. Status Solidi A 203, 1626 (2006).

7. J. Han, J.J. Figiel, M.H. Crawford, M.A. Banas, M.E. Bartram, R.M. Biefeld, Y.K. Song, and A.V. Nurmikko, J. Cryst. Growth 195, 291 (1998). 
8. W.G. Jeong, E.P. Menu, and D.P. Dapkus, Appl. Phys. Lett. 55,244 (1989).

9. M.A. Khan, J.N. Kuznia, R.A. Skogman, D.T. Olson, M.M. Millan, and W.J. Choyke, Appl. Phys. Lett. 61, 2539 (1992).

10. Y. Horikoshi, Semicond. Sci. Technol. 8, 1032 (1993).

11. H.J. Kim, S. Choi, D. Yoo, J.-H. Ryou, R.D. Dupuis, R.F. Dalmau, P. Lu, and Z. Sitar, Appl. Phys. Lett. 93, 022103 (2008).

12. H.J. Kim, S. Choi, D. Yoo, J.-H. Ryou, and R.D. Dupuis, J. Cryst. Growth 310, 4880 (2008).

13. K. Nakano, M. Imura, G. Narita, T. Kitano, Y. Hirosel, N. Fujimoto, N. Okada, T. Kawashima, K. Iida, K. Balakrishnan, M. Tsuda, M. Iwaya, S. Kamiyama, H. Amano, and I. Akasaki, Phys. Status Solidi A 203, 1632 (2006).

14. S. Srinivasan, F. Bertram, A. Bell, F.A. Fonce, S. Tanaka, H. Omiya, and Y. Nakagawa, Appl. Phys. Lett. 80, 550 (2002).

15. C.A. Tran, R.F. Karlicek Jr., M. Schurman, A. Osinsky, V. Merai, Y. Li, I. Eliashevich, M.C. Brown, J. Nering, I. Ferguson, and R. Stall, J. Cryst. Growth 195, 397 (1998).

16. H.J. Kim, Y. Shin, S.-Y. Kwon, H.J. Kim, S. Choi, S. Hong, C.S. Kim, J.-W. Yoon, H. Cheong, and E. Yoon, J. Cryst. Growth 310, 3004 (2008).
17. P. Vennéguès and H. Lahrèche, Appl. Phys. Lett. 77, 4310 (2000).

18. S. Pereira, M.R. Correia, E. Pereira, K.P. O'Donnell, E. Alves, A.D. Sequeira, and N. Franco, Appl. Phys. Lett. 79, 1432 (2001).

19. M.E. Hawkridge, Z. Liliental-Weber, H.J. Kim, S. Choi, D. Yoo, J.-H. Ryou, and R.D. Dupuis, Appl. Phys. Lett. 94, 071905 (2009).

20. N.A. EI-Masry, E.L. Piner, S.X. Liu, and S.M. Bedair, Appl. Phys. Lett. 72, 40 (1998).

21. I.N. Stranski and L. Krastanow, Abhandlungen der Mathematisch-Naturwissenschaftlichen Klasse. Akademie der Wissenschaften und der Literatur in Mainz 146, 797 (1937).

22. P.A. Crozier, M. Catalano, R. Cingolani, and A. Passaseo, Appl. Phys. Lett. 79, 3170 (2001).

23. S.R. Lee, A.F. Wright, M.H. Crawford, G.A. Peterson, J. Han, and R.M. Biefied, Appl. Phys. Lett. 74, 3344 (1999).

24. Q.S. Paduano, D.W. Weyburne, L.O. Bouthillette, S.Q. Wang, and M.N. Alexander, Jpn. J. Appl. Phys. 41, 1936 (2002).

25. S. Choi, H.J. Kim, D. Yoo, J.-H. Ryou, and R.D. Dupuis, J. Cryst. Growth 311, 3252 (2009). 